\title{
Control Systems Integration for Enhanced Vehicle Dynamics
}

\author{
M. Velardocchia and A. Vigliani*
}

Dipartimento di Ingegneria Meccanica e Aerospaziale - Politecnico di Torino, C.so Duca degli Abruzzi 24, 10129

Torino, Italy

\begin{abstract}
This paper deals with improving comfort and handling for a ground vehicle through the coordinated control of different active systems available in passenger cars, e.g., electronic stability control, active roll control and engine torque control.
\end{abstract}

The authors first describe separate control systems, each with its logic, showing advantages and limits, then propose various possible integrations, aiming at exploiting the benefits of a coordinated approach.

Finally, the proposed control logics are tested on a vehicle model: simulation results prove the effectiveness of the approach in improving vehicle response during typical handling maneuvers.

Keywords: Global chassis strategy, control system integration, ESC, ARC, torque vectoring.

\section{INTRODUCTION}

The integration of different chassis control systems can improve vehicle handling, active safety and comfort. Different chassis active systems can interact in various ways to achieve better performances: for example, systems like Torque Vectoring (TV) or Electronic Stability Control (ESC) can be applied to distribute the torque applied to the wheels separately or their intervention can be coordinated, thus leading to enhanced results. Similarly, Active Roll Control (ARC), Active Front Steering (AFS) and Traction Control (TC) can be integrated to increase handling and comfort. The list of active systems goes on and new solutions are continuously under investigation due to development of new sensors and actuators. Emerging technologies devoted to interaction with signals from different vehicles (V2V) and/or from infrastructures (V2I) are promoting new development in the field of chassis active control. Furthermore, chassis systems integration offers a positive impact to improve on vehicle efficiency and saving energy, e.g. integrating active roll-bars with active braking. Positive effects can be determined also by optimizing the drivability, e.g. coordinating powertrain control, active suspensions and active braking.

Many papers are available in literature describing different chassis control systems which are integrated to enhance vehicle performance. A comprehensive state of the art on Integrated Vehicle Dynamics Control (IVDC) is presented by $\mathrm{Yu}$ et al. [1]: some methodologies devoted to IVDC are reviewed and some control strategies to coordinate chassis control systems are summarized; the focus is on integration techniques applied to steering and braking.

*Address correspondence to this author at the Dipartimento di Ingegneria Meccanica e Aerospaziale - Politecnico di Torino, C.so Duca degli Abruzzi 24, 10129 Torino, Italy; Tel: +39.011.0906947;

Fax: +39.011.0906999; E-mail: alessandro.vigliani@polito.it
Yim and Yi [2] present a method for designing an active roll control system, combined with integrated chassis control for hybrid four-wheel-drive vehicles: weighted pseudoinverse-based control allocation and simulation-based optimization are proposed to distribute the control yaw moment. A combination of active front steering with rear torque vectoring actuators in an integrated controller is investigated by Bianchi et al. [3] for vehicle stability and trajectory tracking. An adaptive feedback technique has been used to design the controller.

Stability issues are presented and discussed in depth from a theoretical point of view in [4], where wheeled vehicle behavior is analyzed in terms of longitudinal, lateral and vertical dynamics.

Conte [5] proposes a hybrid controller to increase vehicle lateral stability in critical cornering situations. The controller uses active steering and differential braking as control inputs and consists of three major components: switching logic, reference governor, and transition controller. The switching logic detects critical driving situations and turns on the main controller, which tracks a reference defined by the driver set steering angle.

A Model Predictive Control (MPC) approach for controlling active front steering, active braking and active differentials for autonomous vehicles is presented by Falcone et al. in [6]. At each time step a trajectory is assumed to be known over a finite horizon, and an MPC controller computes the system inputs in order to best follow the desired trajectory on slippery roads at a given entry speed. The MPC problem is based on successive on-line linearization of the nonlinear vehicle model.

Many papers are devoted to control strategies designed to optimize vehicle performance: to this aim, it is necessary to control each tire thereby exploiting the maximum force. Mokhiamar and Abe [7] investigate the effectiveness of weighting coefficient adaptation in simultaneous optimum 
distribution of lateral and longitudinal tire forces to improve vehicle handling and stability. They conclude that weighting coefficient adaptation can exert a large influence on vehicle maneuverability.

Ghoneim et al. [8] describe a stability control algorithm designed using both time-domain and frequency-domain approaches. The control structures include yaw rate feedback design and full-state feedback design based on an estimation of vehicle side-slip velocity.

Jo et al. [9] propose a yaw rate controller designed taking into account steerability, lateral and roll stability, to track the target yaw rate based on sliding mode control theory. Estimators are developed to identify the roll angle and attitude angle of a vehicle based on the simplified roll dynamics model and parameter adaptation approach.

Chu et al. [10] present a combined vehicle dynamics modeling methodology, which can be used to establish a complex model for active roll stability control of ground vehicles. The vehicle model is analyzed by applying this methodology thus simplifying the vehicle dynamic model.

In [11], vehicle comfort and handling improvement issues are dealt with by Poussot-Vassal et al. through the joint control of the suspension and braking systems. Two $H_{\infty}$ gain-scheduled controllers are synthesized to achieve attitude and yaw performances. The proposed strategy tackles the nonlinear tire braking force in an original way and meets the situation dependent objectives of the vehicle in a unified framework.

Zhang et al. [12] present a vehicle yaw stability control strategy devoted to prevent vehicles from spinning and drifting out. The yaw stability control system is integrated with active torque distribution and differential braking system.

Various papers introduce analyses of the enhancements given by the availability of new sensors and estimators to support the improvement of chassis control strategies. With regard to this topic, Gobbi et al. [13] review and present an envisaged improvement of the active safety of road vehicles arising from a real time measurement of forces and moments at the wheels. An original six-component measuring wheel is presented. A proposal of new controls is presented aiming at using the information available and provided by the measuring wheel system. The Authors remark that a considerable improvement of the vehicle active safety can be achieved by sensing the forces at the wheels.

During braking processes, friction plays a key role, as shown by Andrzejewski and Awrejcewicz in [4], where a system with friction is modeled including the governing differential equations. A numerical analysis is carried out, together with an experimental investigation of the physical model introduced. A new idea for the friction pair modeling using both laboratory equipment and numerical simulations is proposed, thus enabling observation and control of the friction force.

Furthermore, Olejnik and Awrejcewicz [14] propose a model to take into account the effects of nonlinearities (stick-slip friction) which may affect automotive control systems: in particular, the paper deals with the numerical simulation of compensation of frictional effects present in a real system designed for observations and experimental estimation of friction force characteristics.

Park et al. [15] describe a methodology to design a model-based estimator to detect impending vehicle rollover. The estimator is designed to obtain good estimates of the vehicle roll angle and roll rate in driving situations in which both maneuvers and road disturbances affect vehicle roll motions. The estimator uses existing sensors, such as the steering wheel angle sensor, lateral acceleration sensor, and yaw rate sensor, on a vehicle equipped with an electronic stability control system. Since road disturbance is unknown or very expensive to measure, disturbance-decoupledobserver design technique is used in the design of the estimator.

Comparisons among performance achievable with different active systems are present in literature: in [16] two chassis control systems are compared with respect to rollover prevention. The first system consists of electronic stability control with differential braking, longitudinal speed control, and continuous damping control. The second system is an active anti-roll bar combined with ESC with differential braking. The capabilities of these systems to control lateral acceleration and roll motion are compared.

In many automotive control design approaches, each active control system is designed and tuned separately, without explicitly considering their interaction. This approach may occasionally lead to conflicting or nonoptimal control strategies, whereas the present work focuses on system integration aiming at minimizing impact on vehicle efficiency.

As an example, the present paper discusses and proposes the use of Active Roll Control (ARC) and Torque Vectoring (TV) instead of Electronic Stability Control (ESC) when possible; in potentially dangerous situations, all the active systems can intervene simultaneously.

These procedures lead to a reduced level of interference with the driver's expectations, i.e., the vehicle does not decelerate during active system intervention thanks to the coordinated logic which acts simultaneously on TVO to increase the engine torque and on ESC (braking) in order to keep the speed constant.

To this aim the proposed integrated control logic is based on the state of the tires: it is necessary to measure or at least estimate the conditions of the tires in terms of forces, longitudinal slip and side slip angle. Examples of measurements of tire forces are reported in [17] and cited references.

The research here presented focuses mainly on the effects of control system integration, so the equations of motion of the vehicle are not reported, since they are very well known and can easily be found in the literature (see, e.g., [18, 19]). The basis for vehicle control are not presented either: detailed information is given in [19].

The present paper initially describes different separate control systems, each with its separate logic, aiming at improving vehicle stability and maneuverability: the first system analyzed is based on torque vectoring; coordinated 
intervention of TVO and ESC is then illustrated and the section ends with the description of an ARC system.

The next section proposes an integration of different active control systems: the goal is to determine the order of activation of the various systems and to illustrate the benefits of a coordinated approach.

Finally, the proposed logics are tested on a vehicle model: simulation results prove the effectiveness of the approach in improving vehicle response during handling maneuvers.

\section{TORQUE VECTORING}

The proposed logic is based on the comparison between the value of the yaw moment $M_{z}^{\text {req }}$ required by the control algorithm and the maximum available value $M_{z}^{\max }$.

In particular, the required moment $M_{z}^{\text {req }}$ is estimated using a sliding mode control [20] aimed at annihilating the difference between the actual and the reference yaw rate, which can be evaluated through a linear vehicle model, e.g., as reported in [21].

\subsection{Estimation of the Available Driving Torque}

It is important noting that there are other active systems able to generate a yaw moment, e.g. Electronic Stability Control (ESC); hence it is necessary to estimate the limits of traction control, since the maximum value $M_{z}^{\max }$ of the torque that can be generated by this control depends on the torque supplied by the engine. To this aim, the value of the engine torque available on the vehicle Controller Area Network (CAN) can be used, though it represents only an estimate, since a direct measure is not available. The information regarding the engaged gear is available on the CAN, so the nominal torque at each differential can be estimated as:

$T_{R R}=T_{R L}=\frac{1}{2} \frac{T_{e}}{\tau\left(1+N_{F R}\right)}$

$T_{F L}=T_{F R}=\frac{1}{2} \frac{N_{F R} T_{e}}{\tau\left(1+N_{F R}\right)}$

where $T_{e}$ is the engine torque, $\tau$ is the total gear ratio (i.e.: $\left.\tau=\omega_{w} / \omega_{e}\right), N_{F R}$ is the ratio between front and rear nominal torques, which is equal to ratio between front and rear vertical forces, and $T_{i j}, i=F$ (front) or $R$ (rear), $j=R$ (right) or $L$ (left), are the torques at each wheel hub.

Obviously, eq.(1) and (2) estimate the torques applied at each wheel without intervention of the electronic differentials.

Assuming that all the available torque is transmitted to the ground (i.e., the tires slip is not too high), the yaw moment $M_{z}$ can be evaluated as

$$
M_{z}=\frac{T_{F R}-T_{F L}}{R_{w}} t_{F} \cos \delta+\frac{T_{R R}-T_{R L}}{R_{w}} t_{R}
$$

where $R_{w}$ is the tire radius, $\delta$ is the steering wheel angle, and $t_{F}$ and $t_{R}$ are the front and rear tracks respectively.

The maximum yaw moment $M_{z}^{\max }$ is obtained when all the torque available at the differential is transmitted to only one wheel of each axle; it holds:

$$
M_{z}^{\max }=\frac{T_{F R}^{\max } t_{F} \cos \delta+T_{R R}^{\max } t_{R}}{R_{w}}
$$

and substituting it follows

$$
M_{z}^{\max }=\frac{T_{e}\left(N_{F R} t_{F} \cos \delta+t_{R}\right)}{\tau R_{w}\left(1+N_{F R}\right)}
$$

which represents the limit of the system, since it is not possible to generate a larger moment without intervening on the accelerator.

\subsection{TVO and ESC Integrated Strategy}

Once $M_{z}^{\max }$ is reached, the system can intervene on the accelerator in order to increase the engine torque. The drawback of this approach is that the speed of the vehicle increases, thus leading to a potentially dangerous situation.

A possible solution is to use the ESC at the same time, aiming at increasing the yaw moment while maintaining constant the total tractive force.

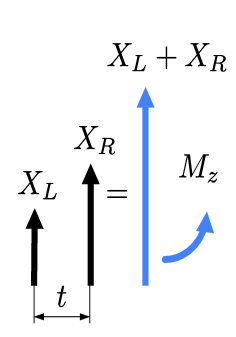

(a)

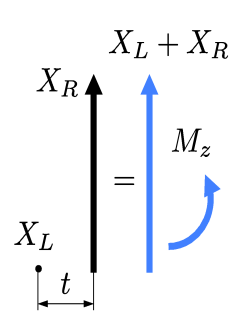

(b)

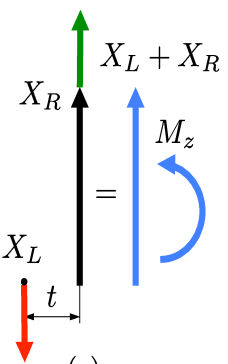

(c)
Fig. (1). longitudinal forces in three different scenarios: (a) TC intervention, (b) maximum TC intervention, (c) maximum TC intervention plus TVO and ESC action.

Fig. (1a) illustrates torque vectoring for traction control: $X_{L}$ and $X_{R}$ are the total longitudinal forces at each side (i.e., the sum of the front and rear forces); consequently $X_{L}+X_{R}$ is the total longitudinal force acting on the vehicle, while $M_{z}$ is the total moment generated by the force distribution.

If a larger moment $M_{z}$ is required, the differentials are able to split the engine torque in order to achieve situation shown in $1(\boldsymbol{b})$, which represents the limit condition: the yaw moment cannot grow further since on the left side the forces are null and they cannot become negative using the differential alone. Therefore, if a greater yaw moment is requested, the system can increase the TVO opening, in order to increase the engine torque, with the consequent undesired growth of the vehicle acceleration. 
The proposed solution is illustrated in Fig. (1c): the additional torque goes only to one side, so that on the left side the total longitudinal force is equal to zero and all the extra force generated by the TVO action is applied on the right side (green arrow). In order to maintain the total longitudinal force almost constant, the ESC intervention is required: by braking the left side of the vehicle is possible to generate a negative force (red arrow) equal to the force generated by the TVO action (green arrow). The consequence is that the total longitudinal force is kept constant while the moment $M_{z}$ increases: in all the three cases shown in Fig. (1), the longitudinal speed of the vehicle is not affected, but the moments about $z$ axle are different.

The procedure can be described by the following condition:

$$
M^{\text {req }}>M_{z}^{\max } \rightarrow \text { combined TVO and ESC intervention. }
$$

Fig. (2) shows the observer and the logic developed for the selection of the most appropriate control action:

- from the top state, the left arrow is followed when only the differential is used, since the requested moment $M^{\text {req }}$ can be generated using the differential alone and consequently neither ESC nor TVO intervention is required;

- when the differential is not sufficient to generate the required moment, the combined intervention of ESC and TVO is requested; the differential continue to supply the maximum torque $M_{d}^{\max }$, computed using the estimator reported in eq.(5), while the remaining part of the moment is provided half by ESC and half by TVO in order to reach the goal without accelerating the vehicle (bottom state of Fig. 2);

- when the previous step is not sufficient to correct the vehicle behavior because also the TVO saturates, the difference between the required and the available yaw moment is supplied by the ESC. Obviously this intervention causes a reduction of the car speed (right state).

\subsection{Tire Saturation}

An optimization algorithm has been developed to compute the torque sent to front and rear axle, so that the tires are used at their best: consequently, the vehicle experiences the maximum acceleration. Despite this control, the additional torque transferred to a wheel could cause the saturation of the corresponding tire; when this occurs, no additional force can be transferred to the ground and consequently the requested $M^{\text {req }}$ cannot be obtained.

Hence, to prevent tire saturation, it is necessary to observe the tire slip. To this aim, a special tire equipped with sensors able to measure road friction would prove extremely useful; nowadays some devices are under development [13]. Other devices, such as the Load Sensing - Hub Bearing Unit

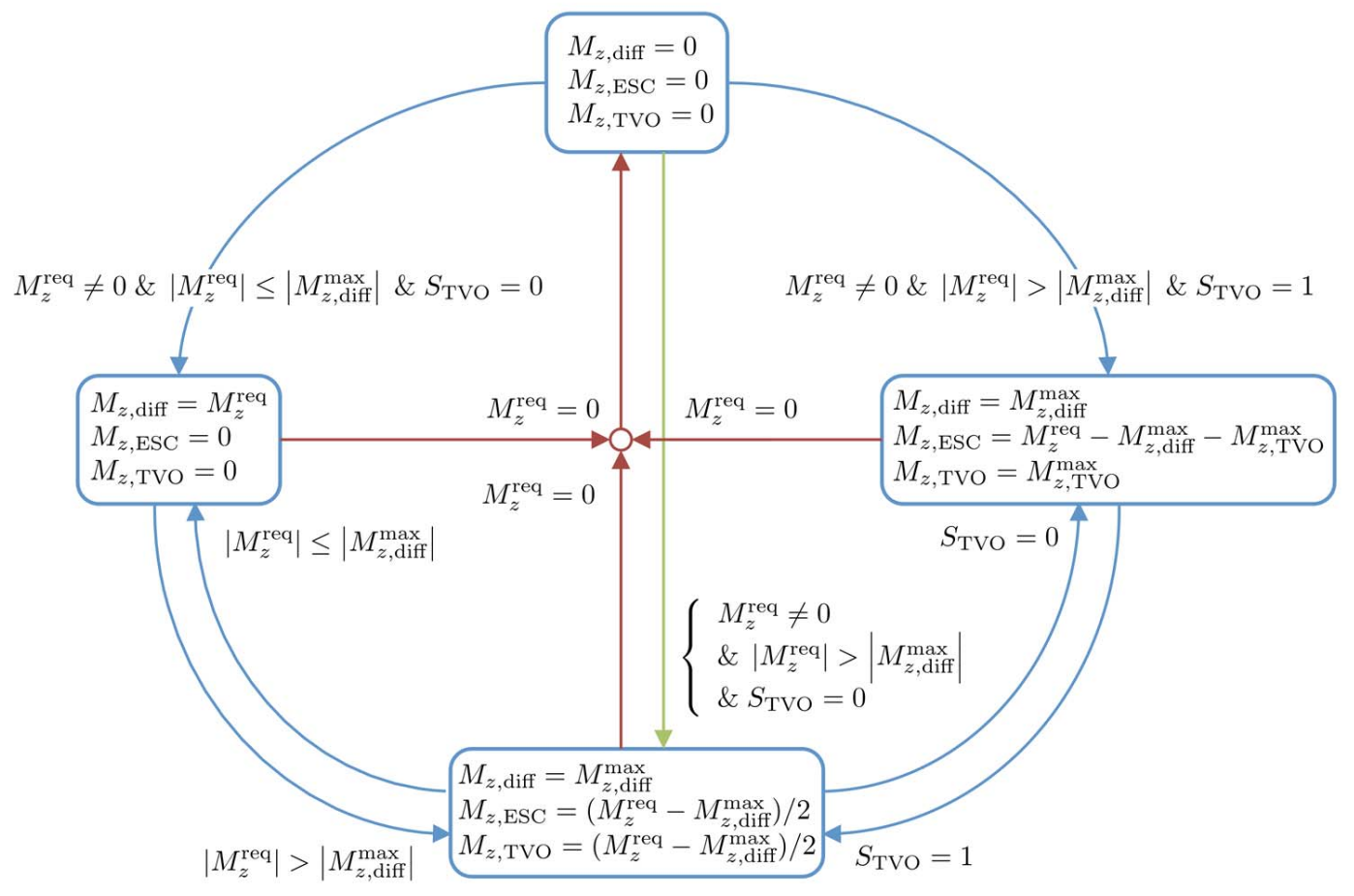

Fig. (2). logic controlling yaw moment $M_{z}$ generation with different active control systems. 


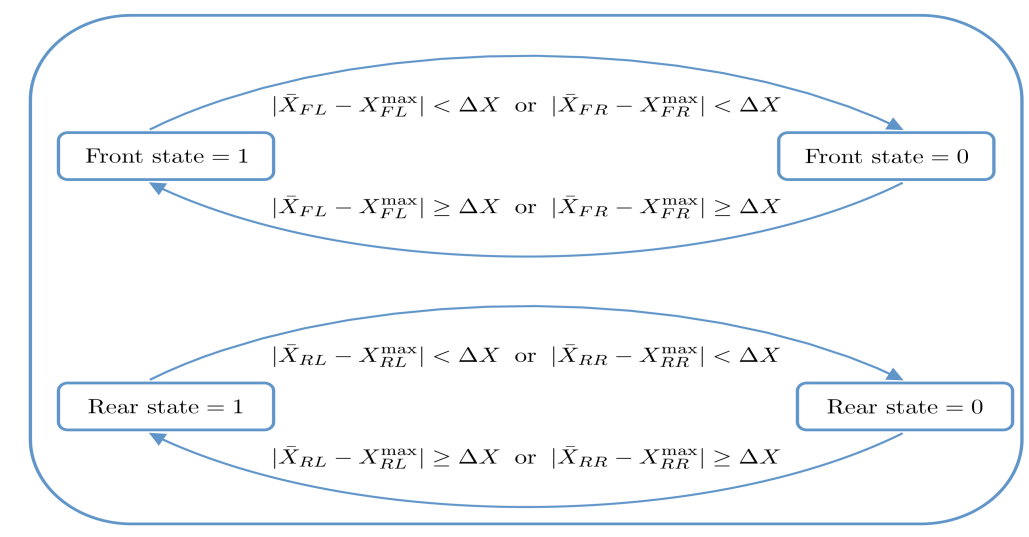

Fig. (3). chart for tire longitudinal force saturation: front and rear wheel are controlled in parallel.

(LS-HBU) [17, 22], permit to measure all the forces exchanged in the hub bearing, in particular their normal and longitudinal components; hence, comparing the maximum potential force of each wheel to the measured value, it is possible to monitor tire saturation. is

The maximum longitudinal force $X$ that can be applied

$X^{\max }=\mu Z$

where $Z$ is the vertical force and $\mu$ is the tire-road friction coefficient. In order to detect saturation in advance, a threshold can be defined, i.e.:

$\mathrm{I} \bar{X}-X^{\max } \mathrm{k}<\Delta X \rightarrow$ saturation

where $\bar{X}$ is the measured value of the longitudinal force.

When condition (8) is true for a tire of one axle, the remaining part of the requested torque is transferred to the other axle and then split between the two sides in order to generate the desired moment. These operations are accomplished by an observer (see Fig. 3) that compares the actual longitudinal forces with the ones estimated using eq.(7).

The proposed force observer has two outputs, one indicating front tire saturation and the other relative to rear wheels. These two signals are directly sent to the chart presented in Fig. (4), whose goal is to select the most suitable action when saturation occurs, choosing between limiting the moment $M_{z}$ available from the differential only or transferring the torque from one axle to the other when a tire of the first axle is in the saturated region.

Three conditions (apart from "No saturation" state) can take place, as illustrated in Fig. (4):
- "F saturation": it occurs when a wheel of the front axle saturates $\left(S_{F}=1\right)$. The logic limits the torque sent to the front axle and consequently the system transfers the remaining torque to the rear differential (F_to_R = 1);

- "R saturation": it occurs when a wheel of the rear axle saturates $\left(S_{R}=1\right)$. In this case, the logic cuts the torque at the rear axle and consequently the system transfers the remaining torque to the front differential (R_to_F = 1);

- "F and R saturation": when at least one tire of both front and rear axles saturates $\left(S_{F}=1\right.$ and $\left.S_{R}=1\right)$, the limit value $M_{z \lim }$ for the torque is reached; then the TVO is reduced to 0 (TVO_cut $=1)$.

\section{ACTIVE ROLL CONTROL}

An alternative way to generate a yaw moment consists in varying the sway bar stiffness by means of an Active Roll Control (ARC) system, based on the dependence of lateral forces on normal load.

In fact, it is possible to obtain the requested moment $M_{z}$ by increasing the force at one axle while decreasing it on the other axle; therefore torque vectoring is designed according to the normal load acting on each axle (Fig. 5).

The yaw moment due to ARC intervention is given by the lateral force variation, i.e.:

$M_{z}=\Delta Y_{F} l_{F}+\Delta Y_{R} l_{R}$

where $\Delta Y_{F}$ and $\Delta Y_{R}$ are the side force $Y$ variations at the front and rear axle, one opposite to the other, $l_{F}$ and $l_{R}$ are the front and rear axle wheelbases. 


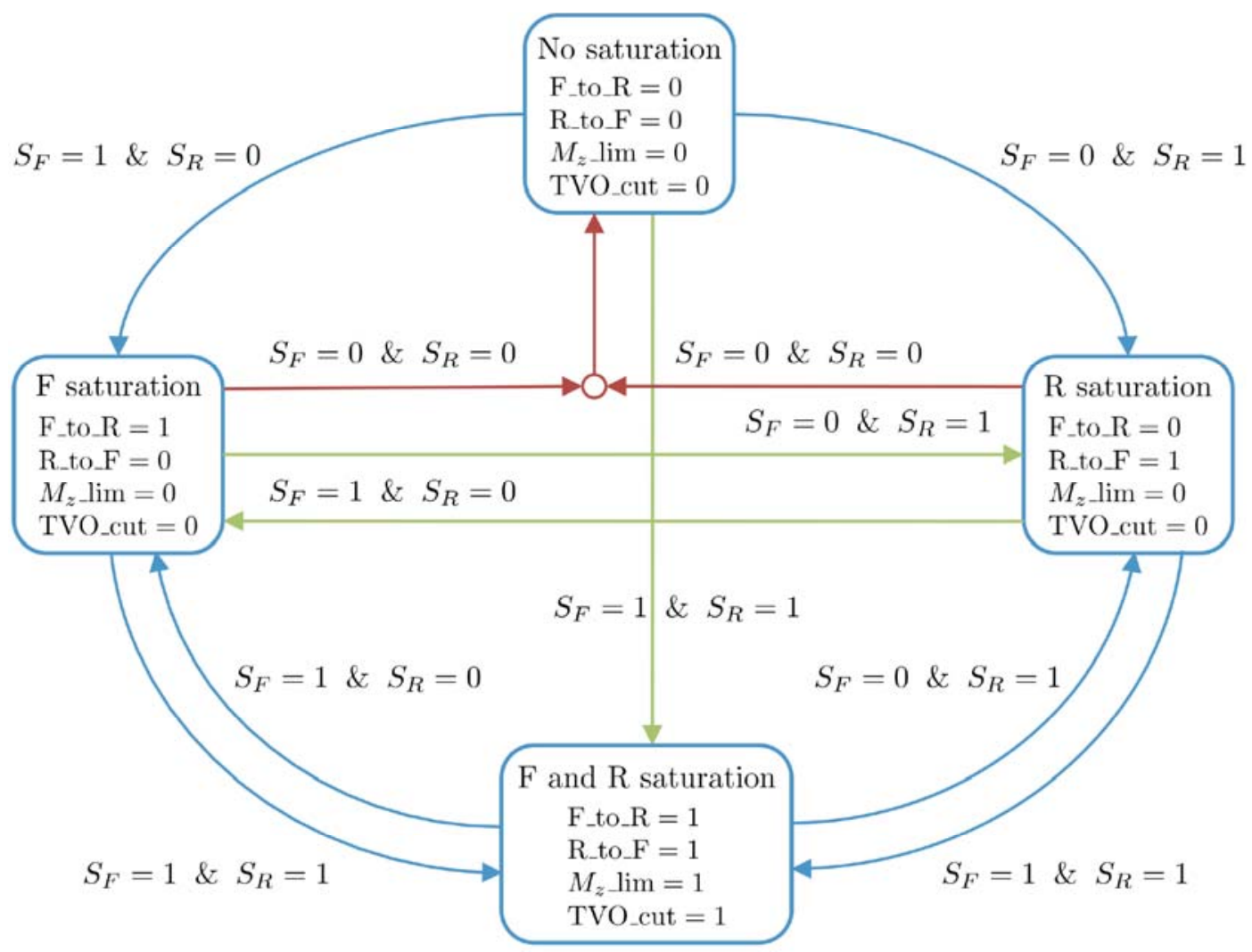

Fig. (4). possible states that can occur when one or both the axles reach longitudinal force saturation.

Because of the dependence of lateral forces on vertical load $Z$, the ratio between front and rear lateral forces can be computed as:

$\frac{\left|\Delta Y_{F}\right|}{\left|\Delta Y_{R}\right|}=\frac{Z_{F L}+Z_{F R}}{Z_{R L}+Z_{R R}}=N_{F R}$

consequently, it holds:

$$
\Delta Y_{R}=\frac{M_{z}}{l_{F} N_{F R}+l_{R}}
$$

$\Delta Y_{F}=N_{F R} \Delta Y_{R}$.

The proposed control strategy uses a proportional control to relate the desired variation of lateral forces with the variation of the sway bar stiffness.

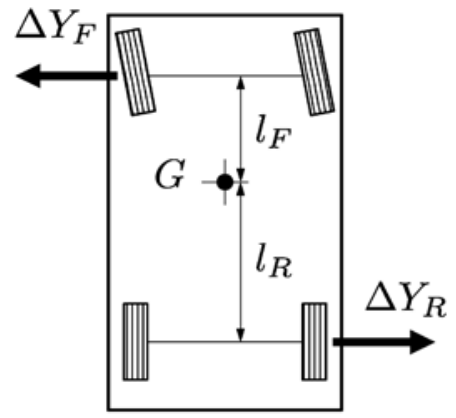

(a)

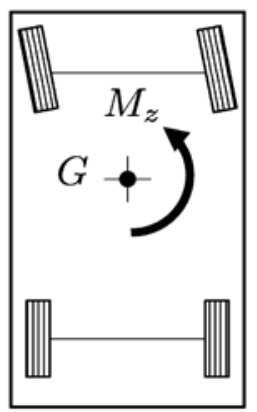

(b)
Fig. (5). Variation of side forces $\Delta Y$ (a) generating the requested moment $M_{z}$ (b).
When the front wheels are steered, it is necessary to take into account the steering wheel angle $\delta$ in order to compute the forces along $y$ direction. Hence, from the tire point of view, the increase of lateral force is

$$
\Delta Y_{\text {tire }}=\frac{\Delta Y_{F}}{\cos \delta} .
$$

Up to this point, the logic does not consider the state of the tires, i.e. if they are close to saturation due to high slip. A possible solution is to consider the behavior of the vehicle correct as long as it is possible to increase the force on one axle. Since the force - slip curve presents a maximum, if the control logic tries to further increase the lateral force after reaching its maximum value, the result would be a smaller force. To avoid this problem, it is necessary to intervene before reaching the tire critical state: the proposed solution consists in comparing the measured force with a reference maximum value. Since this reference value can not be measured, an estimation is performed, e.g., multiplying the normal forces measured by LS-HBU $\bar{Z}$ by the estimated friction coefficient, i.e.:

$|\bar{Y}-\mu \bar{Z}|<\Delta Y \rightarrow$ saturation.

\section{CONTROL SYSTEM INTEGRATION}

The majority of papers dealing with control systems integration faces the topic based on some optimization algorithms [23, 24].

Shimada and Shibahata [25] present a method to determine the best conditions to use a specific control 


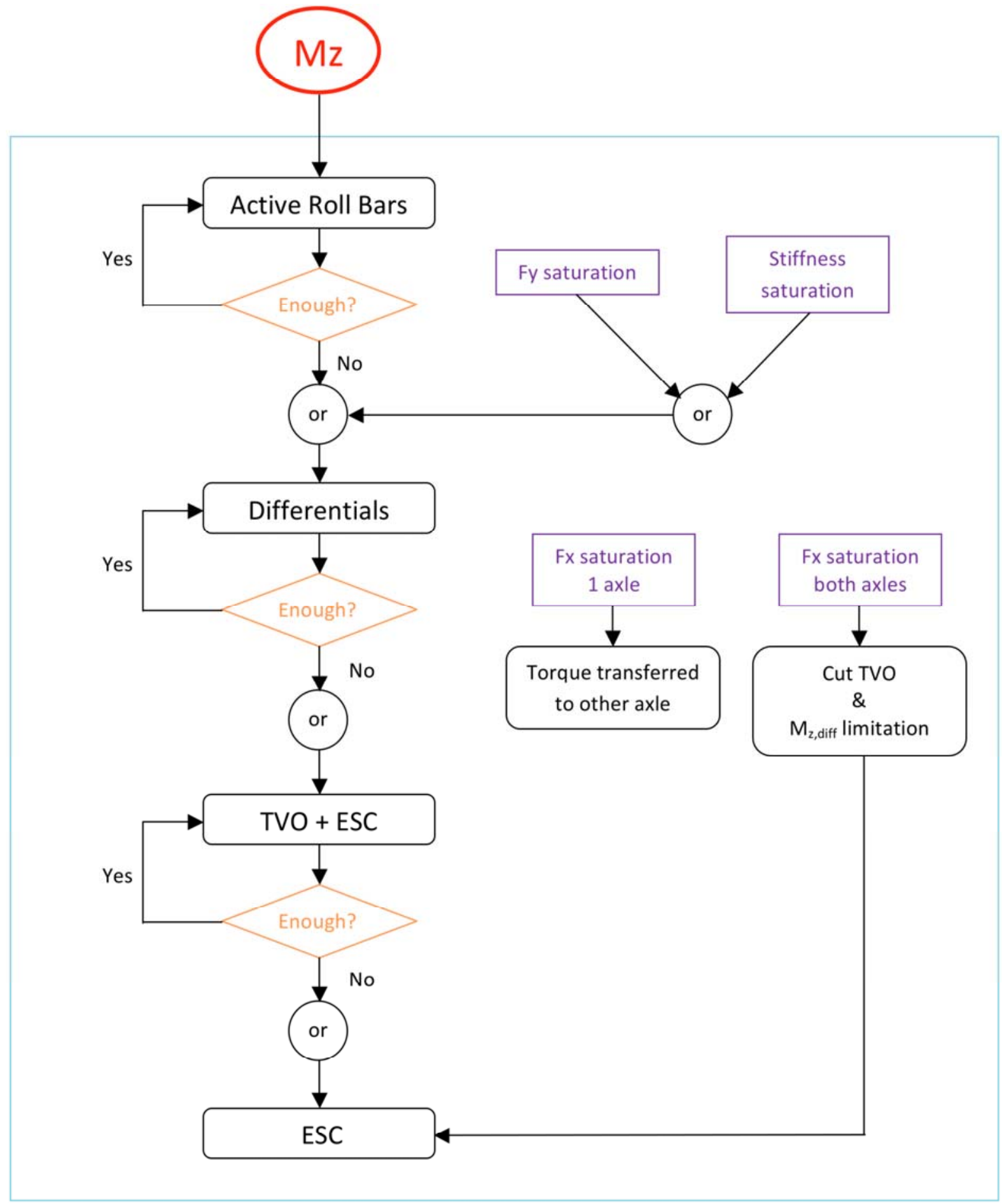

Fig. (6). flow chart describing the integrated control logic.

system, aiming at obtaining the maximum efficiency. Based on $\beta$ method, which uses the variation of the yaw moment with the sideslip angle, it is possible to choose the best system to control the vehicle during acceleration and deceleration; moreover also the effects of active bars, traction control, ESC and active steering are considered. The estimate is performed only during straight motion; consequently this approach is interesting mainly in order to understand the vehicle behavior, but can not be applied as a control logic.
The approach presented in this paper is indeed more practical, since the interest is to develop a control logic that can be directly applied to assess the potential benefits of new sensors, e.g., to understand if the measure of the wheel forces can improve the overall vehicle performance.

\subsection{Control Logic}

The control systems described above should be used in normal driving condition, not only during emergency maneuvers. The idea is to use these systems aiming at an 
"almost-ideal" vehicle, in the sense that it gives the best feeling to the driver and the best efficiency anytime. Hence the vehicle control logic should be able to decide how to intervene considering the advantages and drawbacks of each active system, trying to exploit their best. This statement has many immediate applications: active roll bars allow controlling the vehicle avoiding speed variations or engine interventions, since the action is concentrated on the bars alone; traction control system allows improving the handling performance without braking (as ESC does); finally, ESC allows to achieve the fastest vehicle control and consequently it should always intervene in emergency situations, when it is irrelevant if the vehicle speed is reduced.

Analyzing the properties of the chassis active systems, it appears that roll bars should be used whenever possible and only when their effect becomes negligible the other systems should intervene, with the following order: traction control and then ESC.

Once the order is established, it is necessary to decide the criteria to switch between the control systems. In case the information concerning the road friction is available (e.g., thanks to sensors-equipped tires), it is straightforward to use tires saturation as reference parameter. Moreover, the knowledge of the vertical load on each tire allows to estimate the maximum available longitudinal and lateral forces; the presence of force sensors permits to compare these estimates with the actual values of the forces, thus understanding how much of the potential is used.

The proposed control logic is described in the flow chart of Fig. (6) and is as follows:

- the first active system used is active roll control; then, when lateral forces saturate, i.e. the system has used all its potential, traction control enters into action. It is of interest noting that also bar stiffness has to be considered: when this limit is reached, the system can no longer improve the performance so traction control must intervene even if the tires are far from saturation. In order to detect this limit, a sensor is required in the hydraulic or electrical actuator of the bars;

- $\quad$ active roll bars intervention causes an increase of the lateral force on one axle and a reduction on the other axle; hence only one axle undergoes saturation. The control logic checks only the critical axle to decide if traction control intervention is necessary. In case over-steering is detected, it is necessary to reduce the force at the front axle to "open" the trajectory: thus the front roll bar stiffness has to increase. Consequently, the normal load on the external tire grows and the lateral force at the correspondent tire can reach the maximum: this is the wheel most likely to saturate and so it is the one that should be monitored. Despite this statement, during oversteering maneuvers both tires of the front axle are observed, while during under-steering an opposite situation exists, so the rear axle is constantly checked;

- tires or bars saturation leads to traction control intervention. It is well known that tire longitudinal and lateral force are strongly related [26]. So, if traction control intervenes when the maximum lateral force is reached, only a small part of the longitudinal force is available; consequently, the tire could undergo longitudinal saturation in a very short time. To avoid saturation, the control system requires traction control intervention before the maximum lateral force is reached: a tunable offset between the maximum and the limit is used, so that traction control intervention can be effective.

To obtain the maximum from this control strategy, an algorithm that calculates the state of the tire and is able to detect the approaching of the limit would be the ideal solution: the intervention would be auto-adaptive with the friction coefficient and the tire model; anyway, such algorithm would require some knowledge about the actual tires. In fact, even a simple tire model is necessary, thus implying a dependence on the tire characteristics; consequently, each time the tires are changed, some information should be loaded in the ECU, in order to upgrade the tire model with the new tire data. Nowadays this solution is not applicable, but it could be in the future, when most tires are likely to be equipped with some suitable electronic device.

It is of interest noting that during traction control intervention the active roll bars are still working. While traction control is operating, another check about tire saturation is active; in this case it is important to monitor the state of the longitudinal forces and so the system calculates the theoretical force which should be generated by the tire and compares it with the measured value. If only one tire reaches the limit, an immediate intervention of ESC is not appropriate from an energetic point of view, not only because traction control is not exploited at its maximum, but also because it is more efficient to use the other three tires. Aiming at this, the ECU requires to transfer to the other axle the torque that can not be generated by the axle with the tire close to saturation; obviously this torque has to be split among the tires in order to generate the required moment. Obviously this logic leads to a torque split different from the ideal, but it is more practical and convenient to work with a reduced efficiency than to use ESC.

In case both axles have at least one tire in saturation, the system limits the TVO and a message is sent to the ECU indicating that the limit of the moment $M_{z}$ generated by the differential has been reached reached: when this condition occurs, ESC intervenes.

- only if the combined intervention of TC+TVO is not sufficient because the required moment about $z$ axis is larger than the moment that can be generated, ESC intervenes. Both reduced efficiency and speed reduction are of secondary importance respect to the vehicle dynamic behavior; hence the ECU acts on the brakes: the vehicle behavior is immediately corrected, thus preventing more dangerous situations. The ESC, which may use a closed loop control based on the measured forces (see, e.g., [22]), acts on the brakes in order to supply the difference between the moment generated by the other control systems and the value required by the sliding mode controller. 


\section{SIMULATION RESULTS}

\subsection{ARC}

To understand the potential of the proposed solution, it is of interest observing the effects on handling: Figs. $(\mathbf{7}, \mathbf{8})$ present sideslip angle and yaw rate obtained during a step steer maneuver with constant TVO.

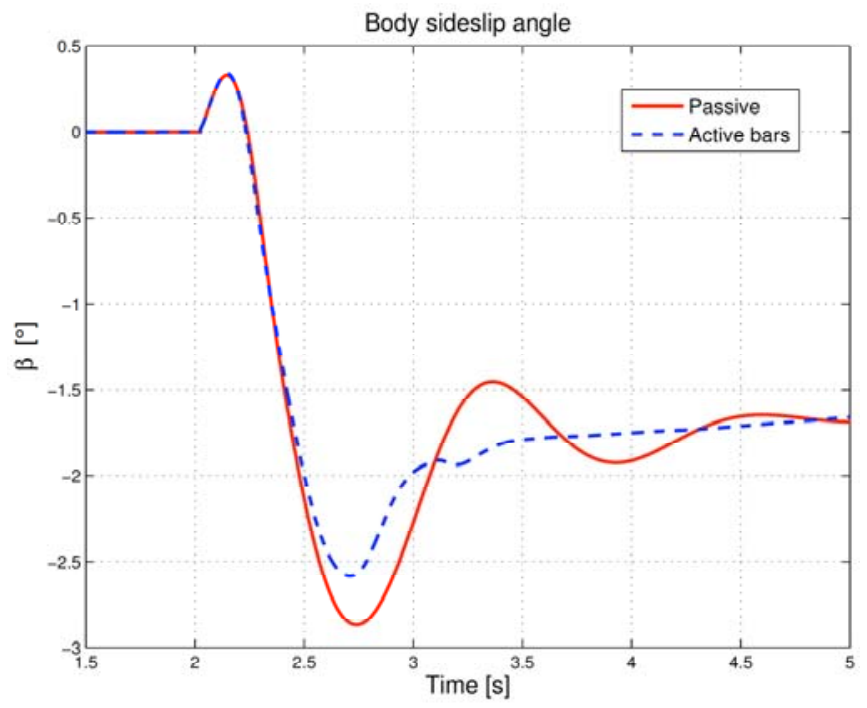

Fig. (7). Body sideslip angle response during step steer: passive vehicle (solid curve) and vehicle with active sway bars (dashed curve).

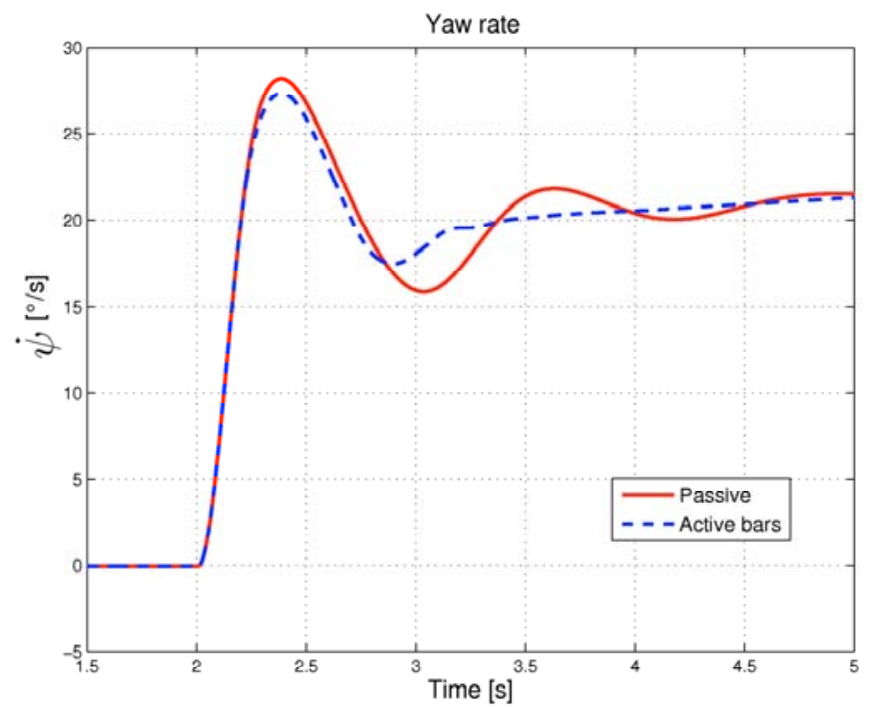

Fig. (8). Yaw rate response during step steer: passive vehicle (solid curve) and vehicle with active sway bars (dashed curve).

Active sway bars are able to correct the vehicle behavior even when used alone. Sliding mode control and response time of the bar actuator do not allow a fast intervention on the car at the beginning of the maneuver, even if some improvement can be noticed also in the initial phase. After the first oscillation this active system proves able to reduce the vibrations and stabilize both sideslip angle and yaw rate.

The vehicle speed does not vary during the combined active systems intervention, since the wheels are not braked (ESC does not intervene).
It is also of interest observing the variation of the requested lateral forces (Fig. 9), i.e., how the total $M_{z}$ is split among the axles.

Fig. (9) also compares the requested forces with "over/under" steering signal: before approximatively $2.1 \mathrm{~s}$, the vehicle presents under-steering behavior and, since it is turning left, an increase of the total front lateral force and a reduction of the rear one is required; between $2.1 \mathrm{~s}$ and 2.75 $\mathrm{s}$ the car is over-steering, then an action with opposite sign is required. In the last part of the simulation, under-steering can be observed again.

These results are obtained increasing the bar stiffness in order to generate a larger antiroll moment. The active roll bars are composed of

- an actuator, generating the active component of the antiroll torque

- the links to the car body, having their own stiffness, generating the passive component of the moment.

Fig. (10) shows active, passive and total roll moment during the same maneuver as in Fig. (9). At the beginning of the maneuver, the front passive roll bar has a correct behavior so that the active bar has only to increase the moment generated. After $2.7 \mathrm{~s}$ a sign correction is required, so the active bar tries to reduce the total moment produced. An opposite behavior can be observed at the rear axle, since forces with opposite direction are required.

From a comfort point of view, active bar intervention causes a reduced feeling, since the roll angle reaches higher values, but the variation is limited and this strategy aims mainly at improving stability, not comfort. Finally, is is of interest noting that, differently than usual, this solution decreases roll stiffness and consequently the suspensions ability to absorb road irregularities improves.

\subsection{Integrated control logic}

The integrated logic has been tested on a complete vehicle model, executing a step steer maneuver with a 100 degrees steer angle at $100 \mathrm{~km} / \mathrm{h}$.

Compared to a passive or ESC vehicle, an "all active" car shows a noticeable improvement in terms of handling characteristics. It can be observed how the integration of the active devices reduces the time response and stabilizes the vehicle faster; moreover the sideslip angle is not affected by oscillations (Fig. 11), so the vehicle gives the driver a better feeling. Finally, yaw rate presents a nearly ideal shape, showing only one oscillation at the beginning of the control intervention (Fig. 12).

Observing vehicle speed (Fig. 13), it is evident the performance improvement due to the proposed control logic, leading to null reduction of velocity. Despite ESC intervention, the simultaneous action on TVO allows to balance the decelerating effect.

Fig. (14) describes the control logic action: with the differentials alone it is possible to generate a moment equal to the thick solid blue curve; if the required moment is larger, an additional torque is supplied by ESC and TVO (the correspondent curves are superimposed: thick dashed and thin solid line). 

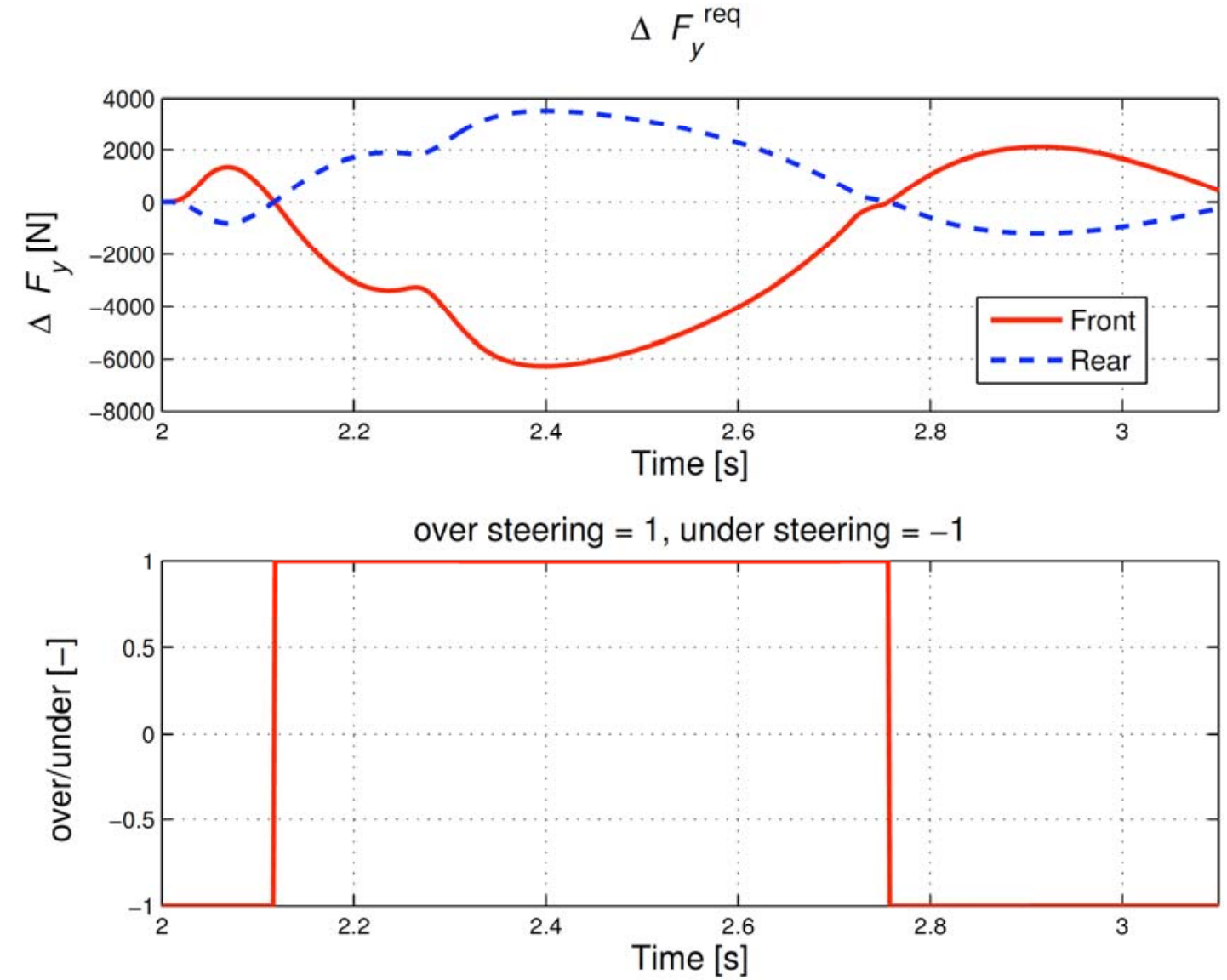

Fig. (9). variation of side forces requested during a step steer maneuver and flag indicating over or under-steering condition.
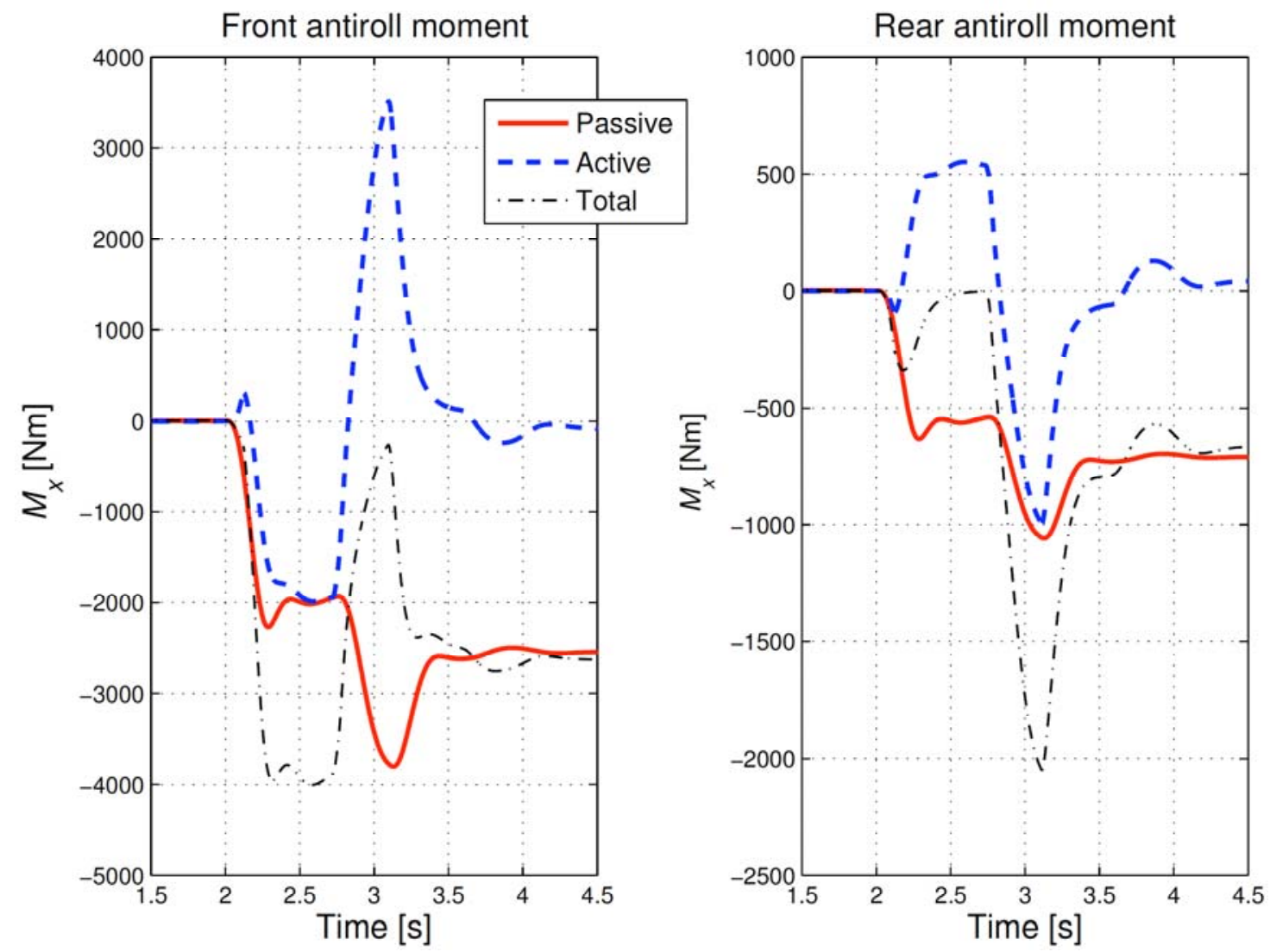

Fig. (10). Antiroll moment generated on front and rear axles by passive and active bars. 


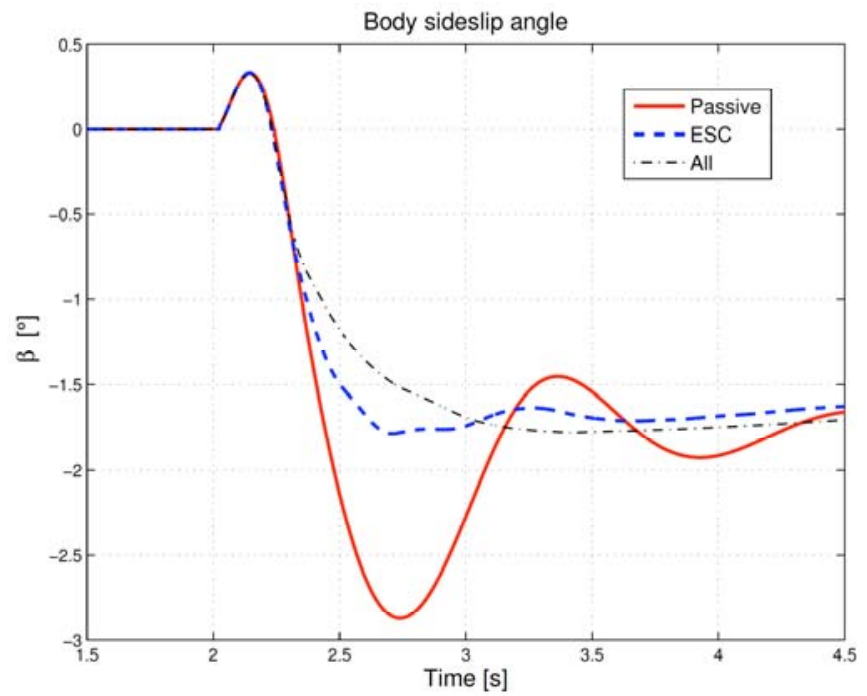

Fig. (11). comparison of body sideslip angle response during step steer among passive vehicle, ESC intervention only and all active system activated.

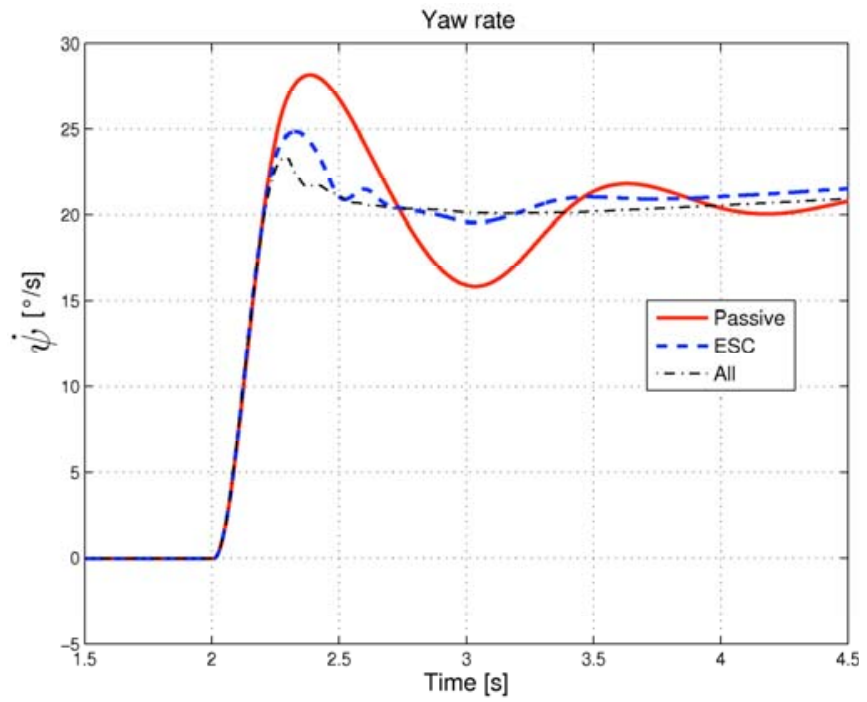

Fig. (12). Comparison of yaw rate response during step steer among passive vehicle, ESC intervention only and all active system activated.

The other main issue of the control logic is the constant check of the state of the tires: if they can not generate the requested force, a different intervention is selected; e.g., when the lateral force can not grow further, active roll bars can not correct the vehicle behavior and consequently the torque request is fulfilled by traction control and ESC.

\section{CONCLUSIONS}

In this paper, a global chassis strategy involving braking systems (ESC), traction control (TC), active roll control (ARC) and active differentials (AD) is presented. The originality of the proposed design lies in the coordinated action of the different active systems, aiming at obtaining the best performance from the vehicle.

Various active systems are discussed: initially the effect on vehicle dynamics is investigated introducing each system one at a time. Then a global control logic which takes advantage of the simultaneous availability of many different active subsystems is presented.

A significant advantage of the proposed approach is that improved performance and vehicle stability can be obtained without exact knowledge of tire force.

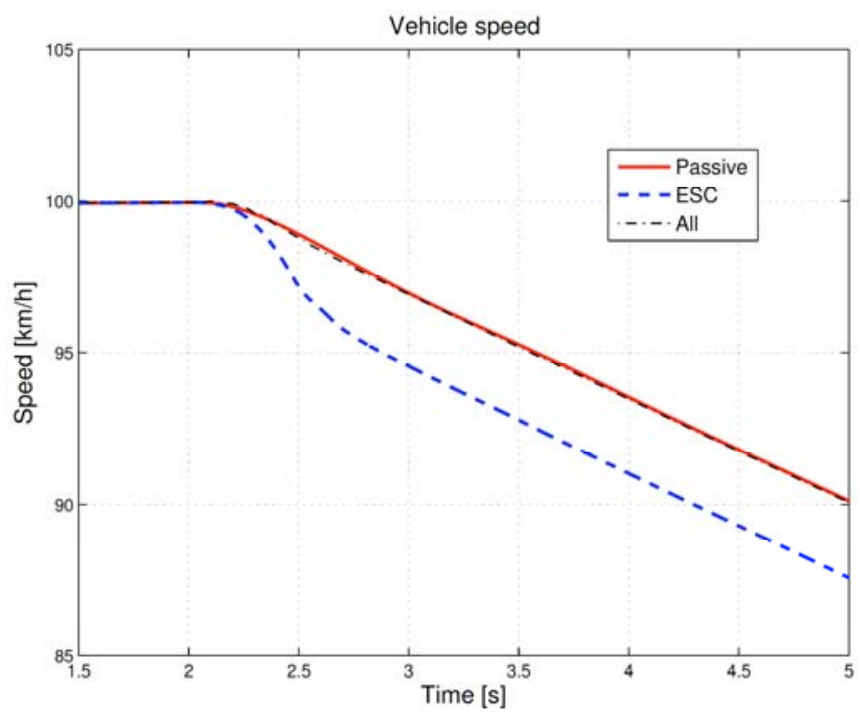

Fig. (13). Vehicle speed is not affected by the activation of "all active" logic since TVO compensates ESC intervention.

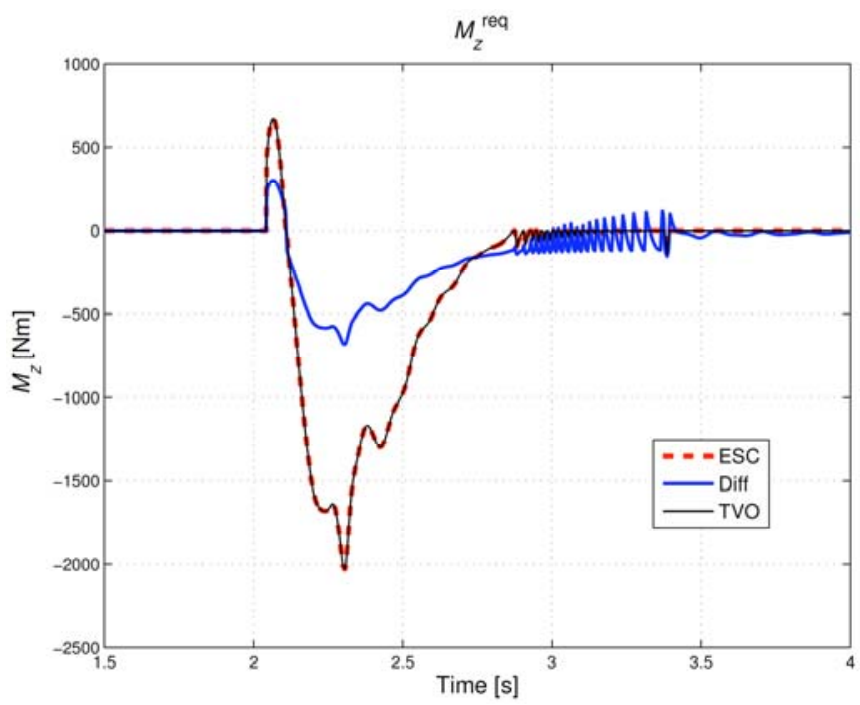

Fig. (14). moment $M_{z}$ generated by different active systems: the differential is not able to supply the moment requested without the coordinated TVO intervention.

Finally, simulations of typical driving situations performed on a nonlinear vehicle model, prove the effectiveness of the proposed approach. The control strategies are tested with numerical simulations, showing substantial improvements in terms of the dynamic response for yaw rate and sideslip angle, thus enhancing car handling and passenger comfort characteristics.

\section{CONFLICT OF INTEREST}

The authors confirm that this article content has no conflict of interest. 


\section{ACKNOWLEDGEMENTS}

Declared none.

\section{REFERENCES}

[1] F. Yu, D.F. Li and D. A. Crolla, "Integrated Vehicle Dynamics Control Đ State-of-the Art Review", in IEEE Vehicle Power and Propulsion Conference, September 2008.

[2] S.Yim and K. Yi, "Design of an active roll control system for hybrid four-wheel-drive vehicles", Proc. Inst. Mechanical Engineers, Part D: Journal of Automobile Engineering, vol. 227, pp. 151-163, February 2013.

[3] D. Bianchi, A. Borri, G. Burgio, M. D. Di Benedetto, and S. Di Gennaro, "Adaptive Integrated Vehicle Control using Active Front Steering and Rear Torque Vectoring", in Joint 48th IEEE Conference on Decision and Control and 28th Chinese Control Conference, December 2009.

[4] R. Andrzejewski and J. Awrejcewicz, Nonlinear Dynamics of a Wheeled Vehicle. Berlin: Springer, 2005.

[5] C. Conte, Hybrid Control of Lateral Vehicle Dynamics, M.S. thesis, Swiss Federal Institute of Technology, Zurich, Switzerland, 2009.

[6] P. Falcone, M. Tufo, F. Borrelli, J. Asgari, and H. E. Tseng, "A Linear Time Varying Model Predictive Control Approach to the Integrated Vehicle Dynamics Control Problem in Autonomous Systems", in Proc. 46th IEEE Conf. on Decision and Control, December 2007.

[7] O. Mokhiamar and M. Abe, "How the four wheels should share forces in an optimum cooperative chassis control", Control Engineering Practice, vol. 12, pp. 295-304, March 2006.

[8] Y.A. Ghoneim, W.C. Lin, D.M. Sidlosky, H.H. Chen, and YuenKwok Chin, "Integrated chassis control system to enhance vehicle stability, vehicle stability", International Journal of Vehicle Design, vol. 23, pp. 124-144, January 2000.

[9] J.S. Jo, S.H. You, J. Y. Joeng, K. I. Lee, and K. Yi, "Vehicle Stability Control System for Enhancing Steerability, Lateral Stability and Roll Stability", International Journal of Automotive Technology, vol. 9, pp. 571-576, October 2008.

[10] D.F. Chu, G.Y. Li, X.Y. Lu, and J. K. Hedrick, "A Combined Dynamics Model for Active Roll Stability Control of Ground Vehicles", in 2nd IEEE International Conference on Computer Engineering and Technology, April 2010.

[11] C. Poussot-Vassal, O. Sename, L. Dugard, P. Gaspar, Z. Szabo, and J. Bokor, "Attitude and handling improvements through gainscheduled suspensions and brakes control", Control Engineering Practice, vol. 19, pp. 252-263, March 2011.
[12] S. Zhang, T. Zhang, and S. Zhou, "Vehicle stability control strategy based on active torque distribution and differential braking", in Proc Int. Conf. on Measuring Technology and Mechatronics Automation, pp. 922-925, April 2009.

[13] M. Gobbi, J. C. Botero, and G. Mastinu, "Improving the active safety of road vehicles by sensing forces and moments at wheels", Vehicle System Dynamics, vol. 46, pp. 957-968, November 2008.

[14] P. Olejnik and J. Awrejcewicz, "Low-speed voltage-input tracking control of a DC-motor numerically modelled by a dynamical system with stick-slip friction", Differential Equations and Dynamical Systems, vol. 21, pp. 3-13, January 2013.

[15] J.I. Park, J.Y. Yoon, D.S. Kim, and K.S. Yi, "Roll state estimator for rollover mitigation control", Proc. Inst. Mechanical Engineers, Part D: Journal of Automobile Engineering, vol. 222, pp. 12891311, August 2008.

[16] S.J. Yim, J.Y. Yoon, W.K. Cho, and K.S. Yi, “An Investigation on Rollover Prevention Systems: Unified Chassis Control versus Electronic Stability Control with Active Anti-Roll Bar", Proc. Inst. Mechanical Engineers, Part D: Journal of Automobile Engineering, vol. 225, pp. 1-14, January 2011.

[17] D. Capra, E. Galvagno, V. Ondrak, B.G. van Leuween, and A. Vigliani, "An ABS control logic based on wheel force measurement", Vehicle System Dynamics, vol. 50, pp. 1779-1796, December 2012.

[18] F. Milliken and D.L. Milliken, Race Car Vehicle Dynamics. Detroit: SAE, 1995.

[19] R. Rajamani, Vehicle Dynamics and Control. New York: Springer, 2012.

[20] C. Edwards and S. K. Spurgeon, Sliding Mode Control: Theory and Applications. London: Taylor \& Francis, 1998.

[21] R. Rajamani, Vehicle dynamics and control. New York: Springer, 2006.

[22] A. Morgando, V. Ondrak, B.G. van Leuween, M. Velardocchia, and A. Vigliani, "An alternative approach to automotive ESC based on measured wheel forces", Vehicle System Dynamics, vol. 49, pp. 1855-1871, December 2011.

[23] E. Esmailzadeh, A. Goodarzi, and G. R. Vossoughi, "Optimal yaw moment control law for improved vehicle handling", Mechatronics, vol. 13, pp. 659-675, September 2003.

[24] P. He and Y. Hori, "Optimum traction force distribution for stability improvement of 4WD EV in critical driving condition", in 9th IEEE International Workshop on Advanced Motion Control, March 2006.

[25] K. Shimada and Y. Shibahata, "Comparison of three active chassis control methods for stabilizing yaw moments", in International SAE Congress, February 1994.

[26] H.B. Pacejka, Tire And Vehicle Dynamics. Oxford: ButterworthHeinemann, 2006.

Received: July 8, 2013

(C) Velardocchia and Vigliani; Licensee Bentham Open.

This is an open access article licensed under the terms of the Creative Commons Attribution Non-Commercial License (http://creativecommons.org/licenses/by-nc/3.0/) which permits unrestricted, non-commercial use, distribution and reproduction in any medium, provided the work is properly cited. 